\title{
Sorovares de Salmonella de origem humana identificados no Estado do Pará, Brasil, no período de 1991 a 2008
}

\author{
Salmonella serovars of human origin identified in Pará State, Brazil from 1991 to 2008
}

Serotipos de Salmonella de origen humano identificados en el Estado de Pará (Brasil) entre 1991 y 2008

Edvaldo Carlos Brito Loureiro

Seção de Bacteriologia e Micologia, Instituto Evandro Chagas/SVS/MS, Ananindeva, Pará, Brasil

\section{Nathalia Danielly Borges Marques}

Seção de Bacteriologia e Micologia, Instituto Evandro Chagas/SVS/MS, Ananindeua, Pará, Brasil

Francisco Lúzio de Paula Ramos

Seção de Bacteriologia e Micologia, Instituto Evandro Chagas/SVS/MS, Ananindeua, Pará, Brasil
Eliane Moura Falavina dos Reis

Laboratório de Enterobactérias, Instituto Oswaldo Cruz, Fundação

Oswaldo Cruz, Ministério da Saúde, Rio de Janeiro, Rio de Janeiro, Brasil

Dália dos Prazeres Rodrigues

Laboratório de Enterobactérias, Instituto Oswaldo Cruz, Fundação

Oswaldo Cruz, Ministério da Saúde, Rio de Janeiro, Rio de Janeiro, Brasil

Ernesto Hofer

Laboratório de Zoonoses Bacterianas, Instituto Oswaldo Cruz, Fundação Oswaldo Cruz, Ministério da Saúde, Rio de Janeiro, Rio de Janeiro, Brasil

\section{RESUMO}

A salmonelose é de distribuição cosmopolita, acometendo todas as faixas etárias, tanto nos países desenvolvidos, como naqueles em desenvolvimento. Este estudo tem como objetivo identificar os sorovares de Salmonella isolados de casos de infecção humana ocorridos em 43 municípios do Estado do Pará no período de 1991 a 2008. Foram utilizadas 890 amostras de Salmonella em coproculturas e hemoculturas, aí incluídos 55 isolamentos de $\mathrm{S}$. Typhi nas fezes e sangue, simultaneamente, de indivíduos sintomáticos. Os casos de infecção por Salmonella foram distribuídos em 13 sorogrupos, com destaque para o grupo O:9 (68,1\%). Foram identificados 47 sorovares de Salmonella, destacando-se S. Typhi $(58,9 \%)$, S. Enteritidis $(5,4 \%)$ e S. Saintpaul $(2,5 \%)$. Ressalta-se a maior prevalência de S. Typhi $(58,9 \%)$ dentre os 47 sorovares identificados, o que demonstra que a febre tifóide representa um sério problema de saúde pública na Região Norte do País, e que necessita de maior atenção dos serviços de saúde quanto à vigilância epidemiológica e ambiental, assim como medidas efetivas para a prevenção e controle.

Palavras-chave: Salmonella; Sorovares; Infecção por Salmonella.

\section{INTRODUÇÃO}

O gênero Salmonella pertence à família Enterobacteriaceae e compreende bacilos Gramnegativos, fermentadores da glicose. A maioria se move por meio de flagelos peritríquios. A classificação atual se baseia em estudos feno e genotípicos, que definiram o gênero em duas espécies: Salmonella enterica, constituída de seis subespécies, e Salmonella bongori ${ }^{14,27}$, obedecendo à seguinte forma de citação: Salmonella enterica subespecie enterica sorovar Typhimurium, ou, simplesmente, Salmonella Typhimurium, com o nome do gênero em itálico e o do sorovar em tipo romano. Na rotina, utiliza-se o esquema de Kauffmann-White na caracterização

\footnotetext{
Edvaldo Carlos Brito Loureiro

Instituto Evandro Chagas

Rodovia BR316, km 7, s/n ${ }^{\circ}$, Levilândia

CEP: 67030-000 Ananindeua-Pará-Brasil

Tel: +55 (91) 3214-2113

E-mail:edvaldoloureiro@iec.pa.gov.br
}

Correspondência / Correspondence / Correspondencia: antigênica de Salmonella, determinando-se as frações dos antígenos somáticos $(\mathrm{O})$ de natureza polissacarídica, e as estruturas flagelares $(H)$, que são de natureza proteica?.

A salmonelose é de distribuição cosmopolita, acometendo todas as faixas etárias, tanto nos países desenvolvidos como naqueles em desenvolvimento, constituindo importante problema de saúde pública. As formas clínicas são representadas por gastrenterite aguda - a mais comum - e febres entéricas (febre tifóide e paratifóide).

Alguns sorovares de Salmonella, como Salmonella Typhi e Salmonella Paratyphi A, B e C, são adaptados exclusivamente ao homem, enquanto que outros sorovares, S. Pullorum, S. Gallinarum, S. Abortusovis e S. Choleraesuis, estão mais adaptados aos animais domésticos ou silvestres, que atuam como fontes de infecção, podendo se propagar à espécie humana ${ }^{13,16,15,18}$. Tal característica define a salmonelose como uma zoonose, além de vincular sua propagação por alimentos, particularmente aqueles ingeridos crus ou mal cozidos ${ }^{20}$. 
Nos Estados Unidos há uma estimativa de que ocorram 1,4 milhões de casos de salmonelose a cada ano ${ }^{6}$, sendo que, na atualidade, os surtos epidêmicos, em sua maioria, estão associados ao sorovar $S$. Enteritidis, com o leite, as carnes e os ovos de galinha como os principais veículos de transmissão ${ }^{2,3,5}$.

No Brasil, as epidemias de enfermidades transmitidas por alimentos, causadas por S. Enteritidis, têm sido registradas em São Paulo ${ }^{1,24}$, Brasília ${ }^{4}, B_{\text {Bumenau }}{ }^{28}$ e Curitiba $^{21}$. No Estado de São Paulo, foram estudados 3.554 casos de Salmonella de origem humana, no período de 1996 a 2003, e se identificaram 68 sorovares, destacando a S. Enteritidis ${ }^{8}$.

Estudos realizados no Estado do Pará, no período de 1975 a 1986, apontam a importância da febre tifóide na Região Norte, onde, no cômputo geral, foram identificados 59 sorovares de Salmonella de infecção humana, com destaque para S. Typhi (14,6\%), seguido de S. Typhimurium (9,6\%), S. Give (9,0\%), S. Agona (7,4\%) e S. Newport $(6,2 \%)^{17}$.

A febre tifóide acomete cerca de 20 a 30 milhões de pessoas nos países em desenvolvimento. A maior incidência está em países da África, Ásia, Caribe e América Central, e América do Sul. No ano 2000 foram estimados mais de 2,16 milhões de casos de febre tifóide em todo o mundo, resultando em 216 mil mortes, sendo que mais de 90\% dos casos ocorreram na Ásia, ${ }^{6,29}$.

No Brasil, a febre tifóide ocorre sob a forma endêmica, com superposição de epidemias, especialmente no norte e nordeste ${ }^{20}$, e está associada a baixos níveis socioeconômicos e de saneamento básico.

Na Amazônia, o Estado do Pará tem registrado a maioria dos casos de febre tifóide. Foram identificados 443 casos de febre tifóide no período de 1987 a 2004, oriundos de diversos municípios do Estado do Pará, incluindo a ocorrência de epidemias em Marabá, Óbidos, Abaetetuba, Moju, Limoeiro do Ajuru e Anajás ${ }^{26}$. Em 1981, no Município de Tucuruí, por ocasião da construção da hidrelétrica de Tucuruí, foi registrado o primeiro surto de febre paratifóide A no Brasil, quando foram identificados 101 casos da doença ${ }^{25}$.

- conhecimento da distribuição geográfica dos sorovares de Salmonella de origem humana é importante para identificar sua incidência/prevalência e o risco à saúde da população, constituindo-se num importante indicador epidemiológico da salmonelose em uma comunidade $^{12}$. No entanto, são escassos os estudos, na Amazônia, que possibilitaram reconhecer os sorovares de Salmonella envolvidos nas infecções humanas e animais e em seus mecanismos de transmissão. Diante do problema, na presente investigação objetivou-se identificar os sorovares de Salmonella isolados de casos de infecções humanas, no período de 1991 a 2008, pela Seção de Bacteriologia do Instituto Evandro Chagas (IEC), oriundos de diferentes municípios do Estado do Pará.

\section{MATERIAIS E MÉTODOS}

\section{AMOSTRAS}

Foram utilizados 890 isolados de Salmonella, que resultaram do processamento de 10.709 coproculturas (663 amostras positivas) e 6.285 hemoculturas (227 amostras positivas) de indivíduos sintomáticos residentes em diferentes áreas do Estado do Pará. A identificação foi realizada na Seção de Bacteriologia do IEC, no período de 1991 a 2008.

\section{COPROCULTURA}

As amostras de fezes naturalmente emitidas foram acondicionadas em frascos apropriados e enviadas ao laboratório, para a realização dos coprocultivos. Em um tempo nunca superior a 2 h após a coleta, as fezes foram semeadas em ágar MacConkey (MC), ágar SalmonellaShigella (SS) e caldo selenito cistina, seguido de plaqueamento em ágar SS, após incubação a $37^{\circ} \mathrm{C}$ por 24 h. As colônias suspeitas (lactose negativas) foram semeadas em ágar tríplice-acúcar-ferro (TSI), seguindo-se a identificação bioquímica, de acordo com as recomendações de Ewing ${ }^{7}$. A confirmação do gênero Salmonella foi realizada pela prova de soroaglutinação, utilizando-se antissoros polivalentes somáticos e flagelares (Difco e Bio-Rad).

\section{HEMOCULTURA}

Logo após a coleta, $10 \mathrm{~mL}$ de sangue periférico foram inoculados em frasco contendo $50 \mathrm{~mL}$ de caldo triptose com fosfato e incubados a $37^{\circ} \mathrm{C}$, observando-se diariamente, por até 15 dias, a presença de crescimento e/ou turvação do meio. Dos frascos que apresentavam turvação, o material era inoculado em ágar SS, após confirmação da presença de bacilos Gram-negativos, por bacterioscopia (coloração de Gram).

\section{IDENTIFICAÇÃO SOROLÓGICA}

A caracterização dos sorovares de Salmonella foi realizada no Laboratório de Enterobactérias do Instituto Oswaldo Cruz/FIOCRUZ/RJ, por detecção dos antígenos somáticos e flagelares, utilizando-se antissoros polivalentes e monovalentes, com ou sem indução de fases flagelares.

\section{RESULTADOS}

A princípio, salienta-se que os 890 isolados de Salmonella resultaram de infecções humanas ocorridas em 43 municípios do Estado do Pará, com destaque para Belém (65,5\%), Abaetetuba (5,7\%), Ananindeua (4,9\%) e Anajás (2,9\%), representando 79\% (704 amostras) do total analisado (Tabela 1).

No cômputo geral, os sorovares de Salmonella identificados foram distribuídos em 13 sorogrupos, sendo que o grupo $\bigcirc: 9$ compreendeu $68,1 \%$ dos isolados, com destaque para os sorovares S. Typhi (492), S. Enteritidis (45) e S. Panama (18) (Tabela 2). 
Tabela 1 - Distribuição das amostras isoladas de Salmonella segundo a procedência geográfica e as fontes de isolamento

\begin{tabular}{|c|c|c|c|c|}
\hline \multirow{3}{*}{ Procedência } & \multicolumn{2}{|c|}{ Origem } & \multirow{2}{*}{\multicolumn{2}{|c|}{ Total }} \\
\hline & \multirow{2}{*}{$\begin{array}{c}\text { Coprocultura } \\
\mathrm{N}^{\circ *}\end{array}$} & \multirow{2}{*}{$\begin{array}{l}\text { Hemocultura } \\
N^{\circ *}\end{array}$} & & \\
\hline & & & $\mathrm{N}^{\circ}$ & $\%$ \\
\hline Belém & 438 & 145 & 583 & 65,5 \\
\hline Abaetetuba & 31 & 20 & 51 & 5,7 \\
\hline Ananindeva & 35 & 9 & 44 & 4,9 \\
\hline Anajás & 16 & 10 & 26 & 2,9 \\
\hline Igarapé-Miri & 17 & 3 & 20 & 2,3 \\
\hline Bagre & 10 & 7 & 17 & 1,9 \\
\hline Parauapebas & 17 & - & 17 & 1,9 \\
\hline Cametá & 11 & 3 & 14 & 1,6 \\
\hline Breves & 8 & 3 & 11 & 1,2 \\
\hline Juruti & 9 & - & 9 & 1,0 \\
\hline Limoeiro do Ajuru & 9 & - & 9 & 1,0 \\
\hline Marituba & 5 & 4 & 9 & 1,0 \\
\hline Moju & 4 & 5 & 9 & 1,0 \\
\hline Acará & 3 & 4 & 7 & 0,8 \\
\hline Portel & 3 & 4 & 7 & 0,8 \\
\hline Barcarena & 6 & - & 6 & 0,7 \\
\hline Curralinho & 2 & 3 & 5 & 0,6 \\
\hline Óbidos & 4 & 1 & 5 & 0,6 \\
\hline Oriximiná & 4 & - & 4 & 0,5 \\
\hline Benevides & 4 & - & 4 & 0,5 \\
\hline Castanhal & 3 & - & 3 & 0,3 \\
\hline Bujaru & 1 & 1 & 2 & 0,2 \\
\hline Marabá & 2 & - & 2 & 0,2 \\
\hline Oeiras do Pará & 2 & - & 2 & 0,2 \\
\hline Ponta de Pedras & 2 & - & 2 & 0,2 \\
\hline Rondon do Pará & 2 & - & 2 & 0,2 \\
\hline Tailândia & 1 & 1 & 2 & 0,2 \\
\hline Viseu & 2 & - & 2 & 0,2 \\
\hline Capanema & - & 1 & 1 & 0,1 \\
\hline Chaves & 1 & - & 1 & 0,1 \\
\hline Colares & - & 1 & 1 & 0,1 \\
\hline Bragança & 1 & - & 1 & 0,1 \\
\hline Gurupá & - & 1 & 1 & 0,1 \\
\hline Irituia & - & 1 & 1 & 0,1 \\
\hline Muaná & 1 & - & 1 & 0,1 \\
\hline Paragominas & 1 & - & 1 & 0,1 \\
\hline Salinópolis & 1 & - & 1 & 0,1 \\
\hline Santarém & 1 & - & 1 & 0,1 \\
\hline Santarém Novo & 1 & - & 1 & 0,1 \\
\hline São João de Pirabas & 1 & - & 1 & 0,1 \\
\hline $\begin{array}{l}\text { São Sebastião da } \\
\text { Boa Vista }\end{array}$ & 1 & - & 1 & 0,1 \\
\hline Tucuruí & 1 & - & 1 & 0,1 \\
\hline Tracuateua & 1 & - & 1 & 0,1 \\
\hline Sem identificação & 1 & - & 1 & 0,1 \\
\hline Total & 663 & 227 & 890 & 99,7 \\
\hline
\end{tabular}

*55 casos de S. Typhi foram isolados das fezes e sangue, simultaneamente;

Sinal convencional utilizado: - Dado numérico igual a zero não resultante de arredondamento.

Convém ressaltar a importância da caracterização dos sorovares de Salmonella, que permitiram o reconhecimento de $492(58,9 \%)$ casos de febre tifóide do total de 835 amostras analisadas no mesmo período.
Tabela 2 - Distribuição numérica dos sorovares de Salmonella identificados e classificados de acordo com os seus sorogrupos, 1991 a 2008

\begin{tabular}{|c|c|c|}
\hline Sorogrupos & Sorovares & $\mathrm{N}^{\circ}$ \\
\hline$O: 2(A) N=2(0,2 \%)$ & S. Paratyphi A & 2 \\
\hline $\begin{array}{c}O: 4(B) \\
N=76(9,1 \%)\end{array}$ & $\begin{array}{l}\text { S. Saintpaul } \\
\text { S. Typhimurium } \\
\text { S. Paratyphi B } \\
\text { S. Agona } \\
\text { S. Schwarzengrund } \\
\text { S. Coeln } \\
\text { S. Derby } \\
\text { S. Chester } \\
\text { S.I } 4,5:-: 1,2^{*} \\
\text { S. Heidelberg } \\
\text { S. Bredeney } \\
\text { Salmonella spp }\end{array}$ & $\begin{array}{r}21 \\
11 \\
4 \\
15 \\
2 \\
2 \\
2 \\
1 \\
2 \\
2 \\
1 \\
13\end{array}$ \\
\hline $\begin{array}{c}O: 7(\mathrm{Cl}) \\
\mathrm{N}=53(6,3 \%)\end{array}$ & $\begin{array}{l}\text { S. Infantis } \\
\text { S. Oranienburg } \\
\text { S. Braenderup } \\
\text { S. Oslo } \\
\text { S. Ohio } \\
\text { S. Isangi } \\
\text { S. Mbandaka } \\
\text { S. Thompson } \\
\text { S.I 6,7:-:-* } \\
\text { Salmonella spp }\end{array}$ & $\begin{array}{r}17 \\
9 \\
5 \\
5 \\
3 \\
2 \\
1 \\
1 \\
2 \\
8\end{array}$ \\
\hline $\begin{array}{c}\mathrm{O}: 8(\mathrm{C} 2-\mathrm{C} 3) \\
\mathrm{N}=29(3,5 \%)\end{array}$ & $\begin{array}{l}\text { S. Newport } \\
\text { S. Corvallis } \\
\text { S. Belém } \\
\text { S. Hadar } \\
\text { S. Albany } \\
\text { S. Muenchen } \\
\text { Salmonella spp }\end{array}$ & $\begin{array}{r}12 \\
7 \\
3 \\
3 \\
1 \\
1 \\
2\end{array}$ \\
\hline $\begin{array}{c}O: 9(\mathrm{D} 1) \\
\mathrm{N}=569(68,1 \%)\end{array}$ & $\begin{array}{l}\text { S. Typhi } \\
\text { S. Enteritidis } \\
\text { S. Panama } \\
\text { S. Miami } \\
\text { S. I 9,12:Iv:-* } \\
\text { Salmonella spp }\end{array}$ & $\begin{array}{r}492 \\
45 \\
18 \\
3 \\
1 \\
10\end{array}$ \\
\hline $\begin{array}{c}O: 3,10(E 1) \\
N=23(2,8 \%)\end{array}$ & $\begin{array}{l}\text { S. Muenster } \\
\text { S. Give } \\
\text { S. Orion } \\
\text { S. Anatum } \\
\text { S.I 3,10:Iv:-* } \\
\text { Salmonella spp }\end{array}$ & $\begin{array}{l}9 \\
3 \\
3 \\
1 \\
1 \\
6\end{array}$ \\
\hline $\begin{array}{l}O: 1,3,19(\mathrm{E} 4) \\
N=3(0,4 \%)\end{array}$ & S. Senftenberg & 3 \\
\hline $\begin{array}{c}O: 11(\mathrm{~F}) \\
\mathrm{N}=4(0,5 \%)\end{array}$ & $\begin{array}{l}\text { S. Rubislaw } \\
\text { Salmonella spp }\end{array}$ & $\begin{array}{l}1 \\
3\end{array}$ \\
\hline $\begin{array}{c}0: 13(\mathrm{G}) \\
N=6(0,7 \%)\end{array}$ & $\begin{array}{l}\text { S. Worthington } \\
\text { S. Poona } \\
\text { Salmonella spp }\end{array}$ & $\begin{array}{l}3 \\
2 \\
1\end{array}$ \\
\hline $\begin{array}{c}\mathrm{O}: 14(\mathrm{H}) \\
\mathrm{N}=4(0,5 \%)\end{array}$ & $\begin{array}{l}\text { S. Carrau } \\
\text { Salmonella spp } \\
\text { S. Gaminara }\end{array}$ & $\begin{array}{l}3 \\
1 \\
3\end{array}$ \\
\hline $\begin{array}{c}O: 16(1) \\
N=5(0,6 \%)\end{array}$ & $\begin{array}{l}\text { S. Brazil } \\
\text { Salmonella spp }\end{array}$ & $\begin{array}{l}1 \\
1\end{array}$ \\
\hline $\begin{array}{c}O: 30(N) \\
N=5(0,6 \%)\end{array}$ & $\begin{array}{l}\text { S. Morehead } \\
\text { S. Urbana }\end{array}$ & $\begin{array}{l}4 \\
1\end{array}$ \\
\hline $\begin{array}{c}O: 38 N=1 \quad(0,1 \%) \\
N=14(1,7 \%) \\
N=3(0,4 \%) \\
N=1(0,1 \%) \\
N=1(0,1 \%) \\
\text { Rugosa, } N=3(0,4 \%) \\
N I^{\dagger} N=33(3,9 \%)\end{array}$ & $\begin{array}{l}\text { S. Freetown } \\
\text { S. Subsp. IV* } \\
\text { S. Subsp. Illa* } \\
\text { S. Subsp. Illb* } \\
\text { S. Subsp. II* } \\
\text { Salmonella spp } \\
\text { Salmonella spp }\end{array}$ & $\begin{array}{r}1 \\
14 \\
3 \\
1 \\
1 \\
3 \\
33\end{array}$ \\
\hline
\end{tabular}

*S.I- S. enterica subsp. enterica; S.II- S. enterica subsp. salamae; S.IllaS. enterica subsp. arizonae; S.Illb- S. enterica subsp. diarizonae; SIV- S. enterica subsp. houtenae; ${ }^{\dagger} \mathrm{NI}$ - não identificado. 


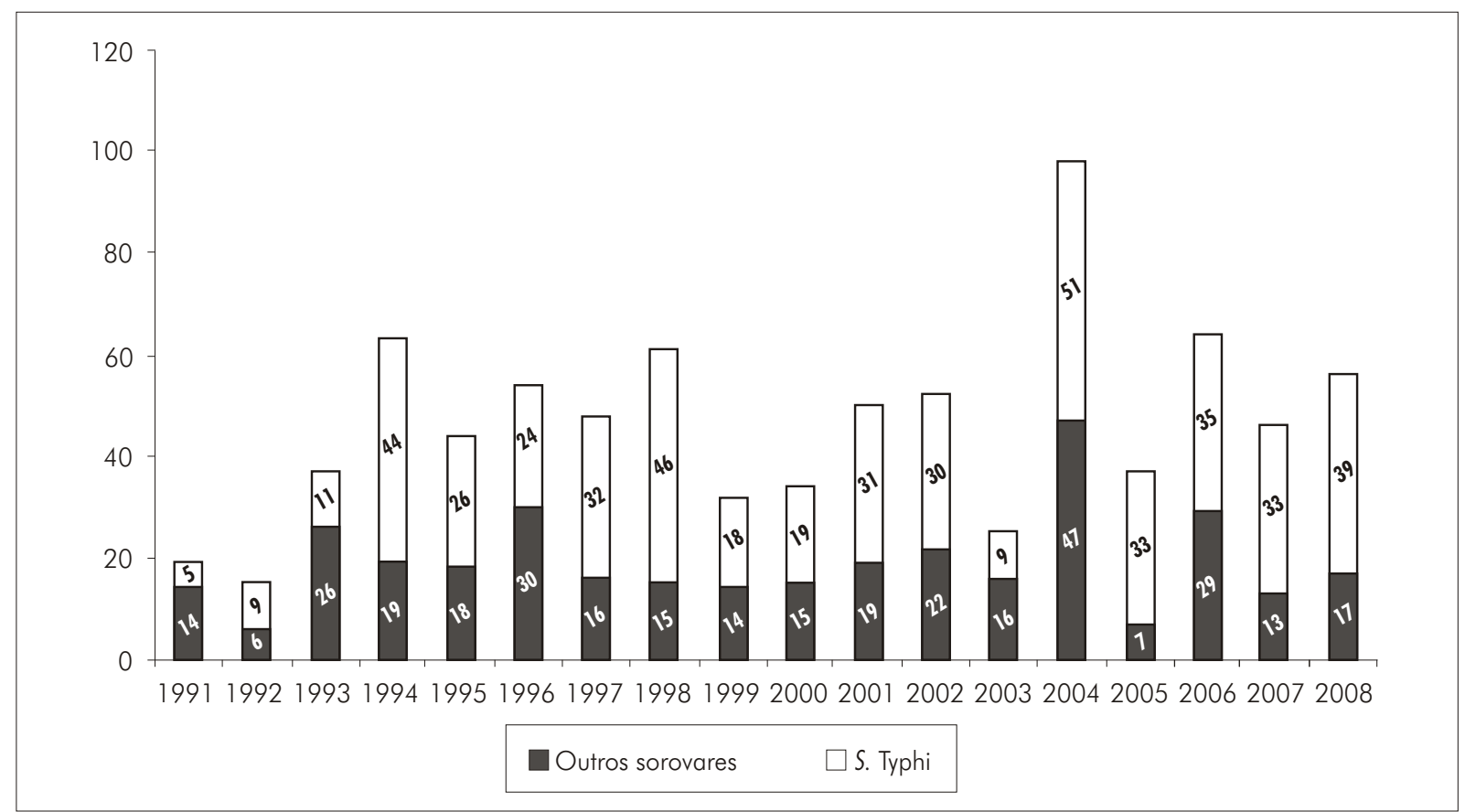

Figura 1 - Distribuição anual numérica de Salmonella spp e S. Typhi isoladas e identificadas no período de 1991 a 2008

Na análise da distribuição anual dos isolados de Salmonella spp no período de 1991 a 2008, destaca-se a maior ocorrência no ano de 2004, com 98 casos, seguido dos anos 2006 (64), 1994 (63) e 1998 (61) (Figura 1). Os casos de febre tifóide ocorreram em todos os anos, e as maiores frequências foram observadas em 2004 (51 casos), 1998 (46), 1994 (44) e 2008 (39), compreendendo $36,6 \%$ do total de casos registrados (Figura 1).

Dentre os casos de febre tifóide, 53,9\% foram detectados na coprocultura, $34,9 \%$ na hemocultura, e $11,2 \%$, tanto na coprocultura como na hemocultura (Tabela 3).

Tabela 3 - Distribuição percentual dos isolamentos de Salmonella Typhi em coproculturas e hemoculturas no período de 1991 a 2008

\begin{tabular}{lrc}
\hline \multicolumn{1}{c}{ Origem } & $\mathrm{N}^{\circ}$ & $\%$ \\
\hline Hemocultura & 172 & 34,9 \\
Coprocultura & 265 & 53,9 \\
Hemocultura e coprocultura & 55 & 11,2 \\
\hline Total & 492 & 100 \\
\hline
\end{tabular}

As amostras analisadas permitiram o reconhecimento de 47 sorovares de Salmonella. Verifica-se nas tabelas 4 e 5 , que S. Typhi (58,9\%), S. Enteritidis $(5,4 \%)$ e S. Saintpaul $(2,5 \%)$, foram os sorovares mais frequentemente detectados no período de 1991 a 2008.

\section{DISCUSSÃO E CONCLUSÃO}

Na vigilância epidemiológica da salmonelose humana é importante o conhecimento dos sorovares prevalentes e/ou incidentes e, também, das vias de transmissão, para que os órgãos de vigilância em saúde intervenham com medidas mais efetivas de prevenção e controle da doença. Ao mesmo tempo, a regularidade na identificação dos sorovares evidencia a introdução de novos tipos sorológicos numa região $0^{12,11}$.

A análise das 835 cepas de Salmonella responsáveis por infecções humanas, no período de 18 anos, demonstrou sua ocorrência em 43 municípios do Estado do Pará, concentrando-se principalmente naqueles com maior contingente populacional, como Belém, com $65,5 \%$ dos isolamentos, seguido de Abaetetuba (5,7\%), Ananindeua (4,9\%) e Anajás (2,9\%) (Tabela 1). Provavelmente as ocorrências decorrem das condições urbanas mais precárias, mas a concentração talvez se deva a que, tendo essas áreas melhores recursos de assistência médica, os diagnósticos e as solicitações de exames laboratoriais são mais frequentes. No geral, dos 47 sorovares de Salmonella identificados (Tabelas 4 e 5), salientam-se a S. Typhi $(65,3 \%)$, S. Enteritidis $(6,0 \%)$ e S. Saintpaul $(2,8 \%)$ como os mais prevalentes. No período de 1999 a 2003, a S. Enteritidis foi identificada em 67,4\% dos casos de infecções gastrintestinais e extraintestinais no Estado de São Paulo, seguida de S. Typhimurium $(5,2 \%)^{8}$. A S. Enteritidis, nos últimos anos, tem sido motivo de preocupação para as autoridades em saúde, pois é o sorovar mais comum em infecções humanas na África, Ásia, Europa e América Latina e Caribe; o sorovar S. Typhi foi o terceiro mais frequente na África e América Latina e Caribe $^{10}$. É interessante notar que, nas regiões analisadas, predominam os sorovares de Salmonella pertencentes ao sorogrupo 0:9 (Tabela 2), compreendendo $68,1 \%$ em confronto com os demais sorogrupos encontrados, ressaltando-se o sorovar $S$. Typhi (58,9\%). 
Tabela 4 - Distribuição dos sorovares de Salmonella identificados no período de 1991 a 1999

\begin{tabular}{|c|c|c|c|c|c|c|c|c|c|}
\hline \multirow{2}{*}{ Sorovar } & \multirow{2}{*}{\multicolumn{8}{|c|}{ Ano }} & \multirow{2}{*}{ Total } \\
\hline & & & & & & & & 1999 & \\
\hline S. Typhi & 5 & 9 & 11 & 44 & 26 & 32 & 46 & 18 & 215 \\
\hline S. Enteritidis & - & - & 3 & 4 & 2 & 5 & - & - & 21 \\
\hline S. Agona & 1 & - & 1 & 4 & - & 3 & 1 & - & 12 \\
\hline S. Infantis & 2 & - & - & 2 & 1 & - & - & 2 & 12 \\
\hline S. Panama & 2 & - & 1 & - & - & 1 & 2 & 1 & 7 \\
\hline S. Newport & - & - & 1 & - & 2 & - & 1 & 1 & 5 \\
\hline S. Typhimurium & - & - & - & - & 1 & 1 & - & 2 & 4 \\
\hline S. Miami & - & - & - & - & - & - & 1 & 2 & 3 \\
\hline S. Ohio & 1 & - & 2 & - & - & - & - & - & 3 \\
\hline S. Oranienburg & 1 & - & 1 & - & - & - & 1 & - & 3 \\
\hline S. Orion & - & - & - & - & - & - & - & - & 3 \\
\hline S. Saintpaul & - & - & - & - & - & - & 2 & - & 3 \\
\hline S. Braenderup & - & - & 2 & - & - & - & - & - & 2 \\
\hline S. Give & 1 & - & - & - & - & 1 & - & - & 2 \\
\hline S. Hadar & - & - & - & - & - & 1 & 1 & - & 2 \\
\hline S. Isangi & 2 & - & - & - & - & - & - & - & 2 \\
\hline S. Muenster & - & - & - & - & 1 & - & - & 1 & 2 \\
\hline S. Paratyphi A & - & - & - & 1 & - & - & - & - & 2 \\
\hline S. Anatum & - & - & - & - & - & - & - & 1 & 1 \\
\hline S. Albany & - & - & - & - & - & - & 1 & - & 1 \\
\hline S. Brazil & - & - & - & - & - & - & - & 1 & 1 \\
\hline S. Coeln & - & - & - & - & - & - & - & 1 & 1 \\
\hline S. Derby & - & - & - & - & - & - & 1 & - & 1 \\
\hline S. Freetown & - & - & - & - & 1 & - & - & - & 1 \\
\hline S. Heidelberg & - & - & - & - & - & - & 1 & - & 1 \\
\hline S. Mbandaka & 1 & - & - & - & - & - & - & - & 1 \\
\hline S. Oslo & - & - & - & - & - & - & - & 1 & 1 \\
\hline S. Poona & - & - & - & - & - & - & 1 & - & 1 \\
\hline S. Thompson & - & - & - & 1 & - & - & - & - & 1 \\
\hline S. Urbana & 1 & - & - & - & - & - & - & - & 1 \\
\hline S. | 4,5:-: 1,2* & - & - & - & - & - & - & 1 & - & 1 \\
\hline S. I 3,10:lv:-* & - & - & - & - & - & 1 & - & - & 1 \\
\hline S. subsp. IV* & - & - & - & - & 1 & 2 & - & 1 & 4 \\
\hline S. subsp. Illa* & - & - & - & - & - & - & - & - & 1 \\
\hline Total & 17 & 9 & 22 & 56 & 35 & 47 & 60 & 32 & 322 \\
\hline
\end{tabular}

Sinal convencional utilizado: - Dado numérico igual a zero não resultante de arredondamento.

Tabela 5 - Distribuição dos sorovares de Salmonella identificados no período de 2000 a 2008

\begin{tabular}{|c|c|c|c|c|c|c|c|c|c|c|}
\hline \multirow{2}{*}{ Sorovar } & \multicolumn{9}{|c|}{ Ano } & \multirow{2}{*}{ Total } \\
\hline & 2000 & 2001 & 2002 & 2003 & 2004 & 2005 & 2006 & 2007 & 2008 & \\
\hline S. Typhi & 19 & 31 & 30 & 9 & 51 & 30 & 35 & 33 & 39 & 277 \\
\hline S. Enteritidis & 1 & 1 & 4 & 5 & 7 & 1 & 2 & - & 3 & 24 \\
\hline S. Saintpaul & 5 & 1 & 1 & 4 & 4 & 1 & - & 1 & 1 & 18 \\
\hline S. Panama & - & 2 & - & - & 2 & - & 1 & 3 & 3 & 11 \\
\hline S. Corvallis & - & - & - & - & 6 & - & 1 & - & - & 7 \\
\hline S. Newport & - & 1 & 1 & - & 2 & - & 1 & 1 & 1 & 7 \\
\hline S. Typhimurium & 2 & 1 & 1 & - & 1 & - & 1 & - & 1 & 7 \\
\hline S. Muenster & - & - & 1 & 1 & 1 & - & 3 & 1 & - & 7 \\
\hline S. Oranienburg & - & - & - & 1 & 3 & - & 1 & - & 1 & 6 \\
\hline S. Infantis & - & 1 & - & - & - & - & 2 & 1 & 1 & 5 \\
\hline S. Oslo & 2 & 1 & - & - & - & - & 1 & - & - & 4 \\
\hline S. Paratyphi B & - & - & 1 & - & - & - & - & - & 3 & 4 \\
\hline S. Agona & - & - & 2 & - & - & - & - & - & 1 & 3 \\
\hline S. Braenderup & - & 1 & - & - & 1 & - & - & - & 1 & 3 \\
\hline S. Belem & 1 & - & - & - & - & - & 2 & - & - & 3 \\
\hline S. Carrau & - & 2 & 1 & - & - & - & - & - & - & 3 \\
\hline S. Gaminara & - & - & - & - & - & - & 2 & 1 & - & 3 \\
\hline S. Senftenberg & - & - & - & 1 & - & - & - & 2 & - & 3 \\
\hline S. Worthington & - & - & - & - & 3 & - & - & - & - & 3 \\
\hline S. Morehead & - & - & 4 & - & - & - & - & - & - & 4 \\
\hline S. Schwarzengrund & - & - & - & 1 & - & - & - & - & 1 & 2 \\
\hline S. 16,7:-:--* & 1 & - & 1 & - & - & - & - & - & - & 2 \\
\hline S. Chester & - & - & - & - & - & - & 1 & - & - & 1 \\
\hline S. Bredeney & - & - & - & - & - & - & - & 1 & - & 1 \\
\hline S. Derby & - & - & - & - & - & - & 1 & - & - & 1 \\
\hline S. Heidelberg & - & - & - & - & 1 & - & - & - & - & 1 \\
\hline S. | 4,5:-: 1,2* & - & 1 & - & - & - & - & - & - & - & 1 \\
\hline S. Hadar & 1 & - & - & - & - & - & - & - & - & 1 \\
\hline S. Muenchen & - & - & - & 1 & - & - & - & - & - & 1 \\
\hline S. 19,12:1v:- & - & - & 1 & - & - & - & - & - & - & 1 \\
\hline S. Coeln & 1 & - & - & - & - & - & - & - & - & 1 \\
\hline S. Give & - & 1 & - & - & - & - & - & - & - & 1 \\
\hline S. Rubislaw & - & 1 & - & - & - & - & - & - & - & 1 \\
\hline S. Poona & - & - & 1 & - & - & - & - & - & - & 1 \\
\hline S. subsp. IV* & - & 1 & - & - & 8 & - & 1 & - & - & 10 \\
\hline S. subsp. Illa* & - & 1 & - & - & 1 & - & - & - & - & 2 \\
\hline S. subsp. Illb* & - & - & - & - & - & - & - & 1 & - & 1 \\
\hline S. subsp. II* & - & - & - & - & - & - & 1 & - & - & 1 \\
\hline Total & 33 & 47 & 49 & 23 & 91 & 32 & 56 & 45 & 56 & 432 \\
\hline
\end{tabular}

S.I- S. enterica subsp. enterica; S.II- S. enterica subsp. salamae; S.Illa- S. enterica subsp. arizonae; S.Illb- S. enterica subsp. diarizonae; SIV- S. enterica

subsp. houtenae;
Sinal convencional utilizado:- Dado numérico igual a zero não resultante de arredondamento. 
No Estado de São Paulo, a S. Typhi representou o quarto sorovar mais frequente e o segundo mais isolado de hemoculturas ${ }^{8}$, enquanto que no Estado do Pará, todos os 227 casos isolados de hemoculturas se caracterizaram como S. Typhi, tendo sido detectados em todos os anos avaliados (Tabelas 4 e 5). É importante registrar a ocorrência destacada no ano de 2004 (Figura 1) quando, dentre os 98 isolados, 51 (52\%) foram resultantes de casos de febre tifóide, distribuídos em 15 municípios do Estado do Pará, com maior frequência de casos em Belém (52,9\%), seguido de Anaiás (13,7\%) e Ananindeua (7,8\%). Toda essa situação retrata a importância do problema da febre tifóide no Estado do Pará, considerando que, dos 835 casos de salmoneloses ocorridos no período de 1991 a 2008 em 43 municípios, a febre tifóide foi diagnosticada laboratorialmente em $34(79,1 \%)$ localidades. Sob esse prisma, salienta-se que a febre tifóide foi endêmica em Belém durante os 18 anos do estudo, e continua sendo, o que sem dúvida alguma, está diretamente relacionado com as precárias condições do saneamento básico e com os baixos níveis de educação da comunidade ${ }^{22}$.

Estes resultados estão de acordo com outros trabalhos realizados anteriormente na região, que demonstraram a alta prevalência de infecção por S. Typhi quando comparada a outros sorovares estudados ${ }^{17,26}$. Nos países desenvolvidos, são relatados poucos casos da infecção tífica, em contraposição às frequências elevadas de febre tifóide em países em desenvolvimento da África e Ásia ${ }^{6,22,23}$.

Os casos de febre tifóide oriundos de demanda espontânea e aqueles encaminhados pelo Sistema Único de Saúde (SUS) compreendem a maioria dos pacientes atendidos no IEC e, no momento do atendimento, apresentam em média 20 a 25 dias de doença ${ }^{26}$. Estes dados podem justificar o maior sucesso de isolamento de S. Typhi em coproculturas $(53,9 \%)$ em relação às hemoculturas $(34,9 \%)$, encontrado no presente estudo (Tabela 3). Contudo, apesar da coprocultura ter apresentado maior índice de positividade na terceira semana de doença, pode-se notar que esse ensaio apresentou índice de positividade substancial durante as duas primeiras semanas e, principalmente, na segunda ${ }^{26}$.

Considerando que o ser humano é o único hospedeiro natural da S. Typhi, as medidas profiláticas devem estar dirigidas para o tratamento de água, saneamento básico, higiene pessoal adequada e educação em saúde, esclarecendo a população sobre a doença e a vacinação, que é uma ferramenta adiciona ${ }^{30}$. Sem dúvida alguma, a implantação e implementação destas medidas possibilitarão minimizar os índices de febre tifóide na Região Amazônica, evitando as perdas econômicas e sociais.

\title{
Salmonella serovars of human origin identified in Pará State, Brazil from 1991 to 2008
}

\begin{abstract}
Salmonellosis presents a cosmopolitan distribution and affects all age groups, not only in developed countries, but also in developing ones. This study aimed to identify the serovars of Salmonella isolated from human infections occurring in 43 areas of Pará State from 1991 to 2008. Eight hundred and ninety samples of Salmonella isolated in coprocultures and blood cultures were analyzed, including 55 isolations of S. Typhi from feces and blood of symptomatic individuals, simultaneously. The cases of Salmonella infections were distributed into 13 serogroups. The majority of them were in group O:9 (68.1\%), and 47 serovars of Salmonella were identified, including S. Typhi (58.9\%), S. Enteritidis (5.4\%) and S. Saintpaul (2.5\%). S. Typhi was the most prevalent (58.9\%) among the 47 identified serovars, which demonstrates that typhoid fever is a serious public health problem in northern Brazil and requires increased attention from health agencies regarding epidemiological and environmental surveillance as effective measures for its prevention and control.
\end{abstract}

Keywords: Salmonella; Serovars; Salmonella Infections.

\section{Serotipos de Salmonella de origen humano identificados en el Estado de Pará (Brasil) entre 1991 y 2008}

\section{RESUMEN}

La salmonelosis es de distribución cosmopolita y afecta a todos los grupos de edad, tanto en países desarrollados como países en desarrollo. Este estudio pretende identificar los serotipos de Salmonella aislados de casos de infección humana entre 1991 y 2008 que se produjeron en 43 municipios del Estado de Pará. Se utilizaron 890 muestras de Salmonella en cultivo de heces y en cultivos de sangre, incluyendo 55 aislamientos de $S$. Typhi en heces y sangre simultáneamente, de individuos sintomáticos. Los casos de infección por Salmonella fueron distribuidos en 13 serogrupos, especialmente para el grupo $0: 9$ (68,1\%). Fueron identificados 47 serotipos de Salmonella, especialmente S. Typhi (58,9\%), S. Enteritidis $(5,4 \%)$ y S. Saintpaul (2,5\%). Destaca la mayor prevalencia de S. Typhi $(58,9 \%)$ entre los 47 serotipos identificados, lo que demuestra que la fiebre tifoidea representa un grave problema de salud pública en la Región Norte de Brasil, y requiere mayor atención de los servicios de salud en lo que se refiere a la vigilancia epidemiológica y ambiental, así como medidas eficaces para la prevención y control.

Palabras clave: Salmonella; Serotipos; Infecciones por Salmonella. 


\section{REFERÊNCIAS}

1 Araújo E, Pacheco MASR, Boni RF, Fonseca YSK, Gelli DS, Fernandes AS, et al. Surtos alimentares por Salmonella Enteritidis associados ao consumo de alimentos à base de ovos, em Sorocaba, SP. Hig Aliment. 1995;9(40):24-6.

2 Badrinath P, Sundkvist T, Mahgoub H, Kent R. An outbreak of Salmonella Enteritidis phage type 34a infection associated with a Chinese restaurant in Suffolk, United Kingdom. BMC Public Health. 2004 Sep;4:40.

3 Braden CR. Salmonella enterica serotype Enteritidis and eggs: a national epidemic in the United States. Clin Infect Dis. 2006 Aug;43(4):512-7.

4 Carmo LS, Vieira AC, Reis JDP, Nascimento RS, Pereira ML, Santos EJ, et al. Staphylococcus aureus and Salmonella Enteritidis present in food implicated in food poisoning. Rev Microbiol. 1996 Apr-Jun;27(2): 122-5.

5 Centers for Disease Control and Prevention. Outbreak of Salmonella serotype Enteritidis infections associated with raw almonds United States and Canada, 20032004. MMWR Morb Mortal Wkly Rep. 2004 Jun; 53(22):484-7.

6 Crump JA, Luby SP, Mintz ED. The global burden of typhoid fever. Bull World Health Organ. 2004 May; 82(5):346-53.

7 Ewing WH. Edward and Ewing's identification of Enterobacteriaceae. 4th ed. New York: Elsevier; 1986. $536 \mathrm{p}$.

8 Fernandes SA, Tavechio AT, Ghilardi AC, Dias AM, Almeida IA, Melo LC. Salmonella serovars isolated from humans in São Paulo State, Brazil, 1996-2003. Rev Inst Med Trop Sao Paulo. 2006 Jul-Aug; 48(4): 179-84.

9 Ferreira EO, Campos LC. Salmonella. In: Trabulsi LR, Alterthum F, editores. Microbiologia. 5. ed. São Paulo: Atheneu; 2008. p. 329-38.

10 Galanis E, Lo Fo Wong DMA, Patrick ME, Binsztein N, Cieslik A, Chalermchaikit $T$, et al. Web-based surveillance and global Salmonella distribution, 20002002. Emerg Infect Dis 2006 Mar; 12(3):381 -8.

11 Hald T, Vose D, Wegener HC, Koupeev T. A Bayesian approach to quantify the contribution of animal-food sources to human salmonellosis. Risk Anal. 2004 Feb;24(1):255-69.

12 Herikstad H, Motariemi Y, Tauxe RV. Salmonella surveillance: a global survey of public health serotyping. Epidemiol Infect. 2002 Aug; 129(1): 1-8.

13 Humphrey T. Public-health aspects of Salmonella infection. In: Wray C, Wray A, editors. Salmonella in domestic animals. Florida: CABI Publishing; 2000. p. 245-63.
14 Le Minor L, Popoff MY. Designation of Salmonella enterica sp. nov., nom. rev., as the type and only species of the genus Salmonella: request for an opinion. Int J Syst Bacteriol. 1987;37:465-8.

15 Lins ZC. Studies on enteric bacteria in the lower Amazon region: II. Salmonella types isolated from wild reptiles in Pará State, Brazil. Rev Microbiol. $1971 ; 2: 165-9$.

16 Lins ZC. Studies on enteric bacterias in the lower Amazon region. I. Serotypes of Salmonella isolated from wild forest animals in Pará State, Brazil. Trans R Soc Trop Med Hyg. 1970;64(3):439-43.

17 Loureiro ECB. Contribuição ao estudo bacteriológico de Salmonella oriundas de diferentes fontes da região Amazônica brasileira. [dissertação]. São Paulo (SP): Universidade de São Paulo, Instituto de Ciências Biomédicas; 1990.

18 Loureiro ECB. Ocorrência do gênero Salmonella em animais silvestres da ordem Edentata, na Região Amazônica, norte do Estado do Pará, Brasil. Rev Latinoam Microbiol. 1985 jan-mar;27(1):31-4.

19 Mead PS, Slutsker L, Dietz V, McCaig LF, Bresee JS, Shapiro C, et al. Food-related illness and death in the United States. Emerg Infect Dis. 1999 Sep-Oct; 5(5):607-25.

20 Ministério da Saúde (BR). Secretaria de Vigilância em Saúde. Guia de vigilância epidemiológica. Brasília; 2005. Febre Tifóide. p. 350-63.

21 Mota CC, Vieira HR, Puzyna IP, Kalache J, Konolsaisen JF, Camargo NL. Toxi-infecção alimentar por Salmonella Enteritidis. Relato de um surto ocorrido em Curitiba - PR, Brasil/julho de 1981. Hig Aliment. 1983;2(3):123-6.

22 Mweu E, English M. Typhoid fever in children in Africa. Trop Med Intern Health. 2008; 13(4):532-40.

23 Ochiai RL, Acosta CJ, Danovaro-Holliday MC, Baiqing D, Bhattacharya SK, Agtini MD, et al. A study of typhoid fever in five Asian countries: disease burden and implications for controls. Bull World Health Organ. 2008;86(4):260-8.

24 Peresi JTM, Almeida IAZC, Lima SI, Marques DF, Rodrigues ECA, Fernandes AS, et al. Surtos de enfermidades transmitidas por alimentos causados por Salmonella Enteritidis. Rev Saúde Pública. 1998 out;32(5):477-83.

25 Pessoa GVA, Lins ZC, Calzada CT, Irino K, Neme SN, Raskin $M$, et al. Identificação e lisotipagem de amostras de Salmonella paratyphi A, causadora de surto epidêmico em Tucuruí, Pará, Brasil, em 1980. Rev Inst Adolfo Lutz. 1983;43(1-2): 105-7.

26 Ramos FL. Febre tifóide: a experiência do Instituto Evandro Chagas. [dissertação]. Belém (PA): Universidade Federal do Pará; 2005. 
27 Reeves MW, Evins GM, Heiba AA, Plikaytis BD, Farmer JJ. Clonal nature of Salmonella typhi and its genetic relatedness to other Salmonellae as shown by multilocus enzyme electrophoresis, and proposal of Salmonella bongori comb. nov. J Clin Microbiol. 1989 Feb;27(2):313-20.

28 Santos SM, Kupek E. Serial outbreaks of food-borne disease in Blumenau, Brazil, caused by Salmonella enteritidis. Braz J Infect Dis. 2000 Dec; 4(6):275-8.
29 Wilde H. Enteric fever due to Salmonella typhi and paratyphi A. A neglected and emerging problem. Vaccine. 2007 Jul;25(29):5246-7.

30 World Health Organization. Background document: the diagnosis, treatment and prevention of typhoid fever. WHO/N\&B/03.07. Geneva; 2003.

Recebido em / Received / Recibido en: 30/7/2009 Aceito em / Accepted / Aceito en: 5/10/2009 